KATHLEEN FLENNIKEN

\title{
My father invented the calendar
}

six days after my mother died.

He sat in her kitchen with visions of boxes

that could contain the house, the wind-tossed apricot blossoms, her phantom voice.

He lined up the boxes in rows of seven and to each column he bestowed a name-

a queue of pretty, indistinguishable nurses, the same brown needle of medicine each time.

By cocktail hour, he'd overwritten them with hair cuts, doctors, and accountants.

He called that day the first, put a slash through the 1 . 